\title{
Cancer: a new role for non-canonical Hippo signaling
}

\author{
Cell Research (2017) 27:459-460. doi:10.1038/cr.2017.27; published online 28 February 2017
}

The molecular mechanisms governing self-renewal and differentiation of the mammary epithelium are incompletely defined; a better understanding of the events implicated in the specification and expansion of luminal progenitors is of particular importance as many breast cancers originate from their transformation. Britschgi et al. found that, in addition to phosphorylating and inactivating YAP, LATS functions as a scaffold to facilitate estrogen receptor- $\alpha$ ubiquitylation by the $\mathrm{E} 3$ ligase CRL4 ${ }^{\mathrm{DCAF} 1}$ and consequently suppresses luminal progenitor specification and expansion.

The ability of cells to differentiate in response to extrinsic and intrinsic signals is the foundation of tissue development. Oncogenic mutations disrupt this process leading to the expansion of cancer stem cells and their aberrantly differentiated progeny [1]. The mammary epithelium forms a network of branched ducts composed of an outer basal layer contacting the basement membrane and an inner luminal layer surrounding a hollow lumen. Much is known regarding the cellular events occurring during prepuberal mammary gland development and lactation. However, although it is known that the estrogen receptor- $\alpha$ $(\mathrm{ER} \alpha)$ drives differentiation of luminal cells [2], the molecular mechanisms governing the specification and expansion of epithelial progenitors are not well defined. Experiments in mouse models and patient-derived breast cancer cells indicate that $B R C A 1$ mutations and HER2 amplifications transform luminal progenitors $[3,4]$. Complex cell fate mechanisms, including dif- ferentiation from a stem cell or transdifferentiation from basal cells, may produce luminal progenitors. Britschgi et al. [5] found that the Hippo pathway kinases LATS $1 / 2$ tightly regulate the fate of primary human breast epithelial stem cells (PHBECs) grown ex vivo. In addition to restraining YAP activation, which drives stem cell self-renewal, LATS1/2 facilitate ER $\alpha$ ubiquitylation by the E3 ligase CRL4 ${ }^{\mathrm{DCAF} 1}$, thereby suppressing luminal progenitor specification and differentiation. Adding to this complexity, NF2/Merlin suppresses the ability of CRL4 ${ }^{\mathrm{DCAF} 1}$ to target LATS, providing a mechanism for activation of YAP in NF2-mutant tumors [6,7].

The authors used a high-content shRNA screen to identify transcripts that suppress proliferation and progenitor marker expression in PHBECs grown as non-adherent mammospheres. The Hippo tumor suppressor pathway kinases LATS $1 / 2$ emerged as potent suppressors of mammosphere formation and luminal differentiation. LATS $1 / 2$ normally restrain the transcriptional module of the Hippo pathway consisting of YAP/TAZ [8]. Notably, transcriptional profiling revealed that the LATS-depleted PHBECs express gene sets enriched in luminal progenitors and mature luminal cells and downregulated in normal basal cells.

The authors show that inactivation of LATS1/2 drives PHBEC 3D growth and luminal fate through two largely independent mechanisms. In addition to promoting YAP/TAZ-mediated transcriptional regulation of targets driving anchorage-independent proliferation, as shown previously $[9,10]$, depletion of LATS1/2 promotes ER $\alpha$ accumulation and activity, driving luminal differentiation. Inducing primary mouse mammary epithelial cells to differentiate along the luminal lineage at the expense of the basal lineage compromises the ability of LATS-depleted cells to repopulate the fat pad, potentially explaining the essential function of LATS during normal gland development [11].

The molecular mechanism connecting LATS to ER $\alpha$ signaling is a new and notable finding. Following the realization that LATS kinase activity is dispensable for $\mathrm{ER} \alpha$ regulation and that ER $\alpha$ appears to be regulated at the protein level, the authors found that ER $\alpha$ ubiquitylation is reduced by LATS depletion. These findings suggest that LATS promotes ER $\alpha$ ubiquitylation and subsequent proteasomal degradation. Quantitative proteomics and immunoprecipitation experiments revealed that ER $\alpha$ exists in a complex with DCAF1, DCAF13, and LATS1. Although the role of DCAF13 in this complex remains undefined, LATS1 interacts with and recruits ER $\alpha$ to CRL4 ${ }^{\text {DCAFl }}$ for ubiquitylation. Indeed, depletion of LATS1/2 reduces ER $\alpha$ ubiquitylation, which depends on CRL4 $4^{\mathrm{DCAF} 1}$. Consistently, LATS1 overexpression decreases and conversely, DCAF1 depletion increases $\mathrm{ER} \alpha$ levels in cell lines. In light of prior evidence indicating that Merlin suppresses the ability of CRL4 ${ }^{\mathrm{DCAF} 1}$ to ubiquitylate LATS1 and target it for degradation [7], it is tempting to speculate that inactivation of Merlin, which occurs in a small fraction of breast cancers [12], may lead to activation of YAP and stabilization of ER $\alpha$. On the other hand, CRL4 ${ }^{\mathrm{DCAF}}$-mediated mono- and oligoubiquitylation of LATS2 inhibits kinase 


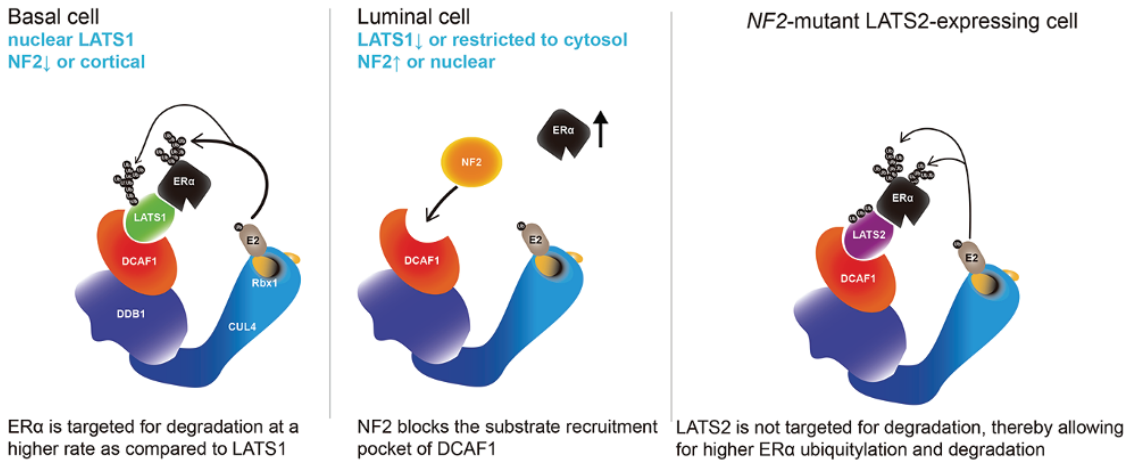

Figure $1 \mathrm{CRL} 4^{\mathrm{DCAF} 1}$-mediated ER $\alpha$ degradation in different cellular contexts.

activity in lieu of degradation. Thus, it is anticipated that in NF2-mutant cells expressing predominantly LATS2, ER $\alpha$ degradation would be promoted, leading to the expansion of more immature cells (Figure 1).

The authors' finding that LATS1 expression is downregulated in a large fraction of breast cancer cell lines and samples is of therapeutic interest. Consistent with the findings that LATS1 promotes ER $\alpha$ degradation, high expression of LATS1 correlated with low ER $\alpha$ expression in breast cancer samples. Notably, LATS1 depletion significantly reduced the sensitivity of $\mathrm{ER}^{+}$cells to Fulvestrant, a selective estrogen receptor degrader (SERD) thought to function by binding to and destabilizing ERs via the ubiquitin-proteasome system. The authors found that low LATS $1 / 2$ expression is associated with reduced relapsefree survival of breast cancer patients treated with anti-estrogen therapy (such as Fulvestrant), but not patients treated with tamoxifen alone. Unlike SERDs, tamoxifen is a canonical ER antagonist. These data strongly suggest that loss or reduced expression of LATS1/2 drives SERD therapeutic resistance by abrogating an innate ER $\alpha$ degradation mechanism. In ER $\alpha$-positive breast cancers with low LATS1/2, tamoxifen therapy may help to overcome SERD resistance.

These findings reveal that LATS $1 / 2$ play an important role in regulating luminal differentiation. Notably, LATS1 is cytoplasmic in luminal cells, which may prevent it from facilitating ER $\alpha$ degradation. Britschgi et al. [5] speculate that cytosolic LATS can suppress YAP/ TAZ activity in luminal cells, although previous findings have indicated that LATS1/2 are predominantly active in the nucleus [7]. Further work is needed to fully understand in which subcellular compartment LATS1/2 are activated and target YAP in mammary stem cells and their progeny. When LATS $1 / 2$ are lial cells, ectopic ER $\alpha$ activity drives luminal differentiation from bipotential progenitors or trans-differentiation from basal cells. While increased YAP/TAZ activity fosters anchorage-independent growth of luminal and luminal progenitor cells in this context, it probably has marginal effects in basal cells since YAP/TAZ activity may be at a saturatdepleted in primary mammary epithe- ing level. In the context of disease, these findings reinforce the concept that reduced expression of LATS1/2 may increase the stemness and invasiveness of luminal mammary carcinomas via dysregulation of YAP/TAZ $[9,10]$. Most notably, they provide a new mechanism for SERD resistance and suggest that agents targeting CRL4 ${ }^{\mathrm{DCAF}}$ may overcome such resistance.

Jonathan Cooper ${ }^{1}$, Filippo G Giancotti ${ }^{2}$

${ }^{1}$ Research and Early Development, Genentech Inc., 1 DNA Way, South San Francisco, CA 94080, USA; '2Department of Cancer Biology and David H Koch Center for Applied Research of Genitourinary Cancers, UT MD Anderson Cancer Center, Houston, TX 77230, USA

Correspondence: Jonathan Coopera ${ }^{\mathrm{a}}$, Filippo G Giancotti ${ }^{b}$

aE-mail: cooper.jonathan@gene.com

bE-mail: FGGiancotti@MDAnderson.org

\section{References}

1 Giancotti FG. FEBS Lett 2014; 588:25582570.

2 Gjorevski N, Nelson CM. Nat Rev Mol Cell Biol 2011; 12:581-593.

3 Lim E, Vaillant F, Wu D, et al. Nat Med 2009; 15:907-913.

4 Vaillant F, Asselin-Labat ML, Shackleton M, et al. Cancer Res 2008; 68:7711-7.

5 Britschgi A, Duss S, Kim S, et al. Nature 2017; 541:541-545.

6 Li W, You L, Cooper J, et al. Cell 2010; 140:477-490.

7 Li W, Cooper J, Zhou L, et al. Cancer Cell 2014; 26:48-60.

8 Meng Z, Moroishi T, Guan KL. Genes Dev 2016; 30:1-17.

9 Chan SW, Lim CJ, Guo K, et al. Cancer Res 2008; 68:2592-2598.

10 Overholtzer M, Zhang J, Smolen GA, et al. Proc Natl Acad Sci USA 2006; 103:1240512410.

11 Curtis C, Shah SP, Chin SF, et al. Nature 2012; 486:346-352.

12 St John MA, Tao W, Fei X, et al. Nat Genet 1999; 21:182-186. 